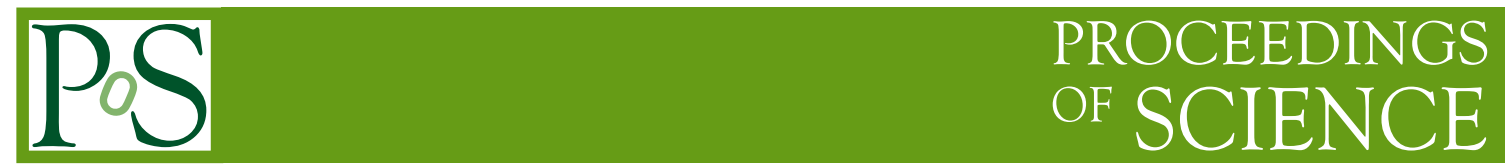

\title{
Searches for vector-like quarks with top quarks
}

\author{
Jeff Tseng* \\ University of Oxford \\ On behalf of the ATLAS and CMS Collaborations. \\ E-mail: jeff.tseng@physics.ox.ac.uk
}

\begin{abstract}
Searches for vector-like quarks at the ATLAS and CMS experiments in approximately $20 \mathrm{fb}^{-1}$ of $8 \mathrm{TeV}$ data from the LHC are presented. No significant excesses are reported, but analysis techniques developed in these searches are expected to play a role in increasing sensitivity in upcoming $13 \mathrm{TeV}$ searches.
\end{abstract}

8th International Workshop on Top Quark Physics

14-18 September, 2015

Ischia, Italy

\footnotetext{
* Speaker.
} 


\section{Introduction}

Vector-like quarks (VLQ's), fermions for which left-handed and right-handed fields transform in the same way under Standard Model gauge groups, are an attractive generic signature that arise in a number of models of physics beyond the Standard Model. The theory is described elsewhere in these proceedings, in particular by M. Peskin and A. Deandrea.

This article describes recent searches for VLQ's conducted by the ATLAS [1] and CMS [2] experiments at the Large Hadron Collider [3] operating at $\sqrt{s}=8 \mathrm{TeV}$. We denote the vector-like top partner with charge $+\frac{2}{3} e$ as $T$, and the bottom partner with charge $-\frac{1}{3} e$ as $B$. More exotically charged partners, $T_{5 / 3}$ and $B_{4 / 3}$, arise in some models. Assuming that these new particles couple primarily to third-generation quarks $t$ and $b$, the most important decay channels include the $T \rightarrow b W$ and $B \rightarrow t W$ which may be expected for Standard Model-like chiral fourth-generation quarks, but also the flavor-changing neutral current decays $T \rightarrow t Z / H$ and $B \rightarrow b Z / H$. Different models give different branching fractions for these modes. While most searches assume pair production, it is expected that as mass limits are pushed higher, electroweak production of single VLQ's will become more important. Searches have also been conducted for decays of VLQ's of unspecified flavor $Q$ to lighter-generation Standard Model quarks $q$. Due to space limitations, this article will focus on the most recent results and note developments in analysis techniques. In all the analyses touched upon below, readers are referred to the publications for full details.

\section{Pair production with leptonic signatures}

Both the ATLAS and CMS experiments have published a number of searches for pair-produced VLQ's where at least one of the daughter particles decays further to at least one isolated high- $p_{\mathrm{T}}$ lepton, usually an electron or muon. These leptons are expected to be accompanied by a number of high- $p_{\mathrm{T}}$ jets, some of which will be $b$-tagged, and gauge and Higgs bosons, depending on the particular VLQ and decay channel. The CMS experiment has consolidated its results into two papers, one focused on $B \bar{B}$ searches [4], and a more recent one focused on $T \bar{T}$ searches [5]. Both papers update and finalize previous analyses with the full $19.7 \mathrm{fb}^{-1}$ dataset at $8 \mathrm{TeV}$, and calculate limits based on the combination of the analyses, taking into account correlated and uncorrelated systematic uncertainties. The ATLAS experiment, on the other hand, has published signaturebased analyses on $20.3 \mathrm{fb}^{-1}$ of data: single lepton+jets [6, 7], opposite-sign dileptonic $\mathrm{Zt} / b$ [8], same-sign dileptons with $b$ quarks [9], and leptonic $W$ with a light quark [10].

The ATLAS single-lepton search for $B \bar{B} \rightarrow t W+X$ [6] illustrates a typical analysis strategy: Fig. 1(left) shows unit-area-normalized Monte Carlo distributions of $H_{\mathrm{T}}$, which is defined as the scalar sum of the $p_{\mathrm{T}}$ 's of the lepton, jets, and missing $p_{\mathrm{T}}$, for the signal and the most important backgrounds, in this case $t \bar{t}$ and $W+$ jets production. A clear separation is seen, and this variable is the most important single discriminant in this and other analyses (where $H_{\mathrm{T}}$ may sum over different ranges of objects, and is sometimes denoted $S_{\mathrm{T}}$ ). More information, however, exists in other variables such as the angular separation between the lepton and the tagged $b$ jet, and the transverse mass of the lepton and missing $p_{\mathrm{T}}$ system. In this analysis, this information is combined into a single discriminant using a Boosted Decision Tree (BDT) [11]. As shown in Fig. 1(right), the data distribution for the BDT discriminant is consistent with the background prediction from 
a combination of Monte Carlo and data-driven methods and validated in background-rich control regions. Limits for $B \bar{B}$ production are calculated as a function of $B$ branching fractions to $t W, b Z$, and $b H$, and are shown combined with other ATLAS analyses in Sec. 5. This analysis can also be interpreted as a search for pair production of $T_{5 / 3}$ fermions. CMS has used a BDT in its $T \bar{T}$ search in single-lepton data $[12,13]$.
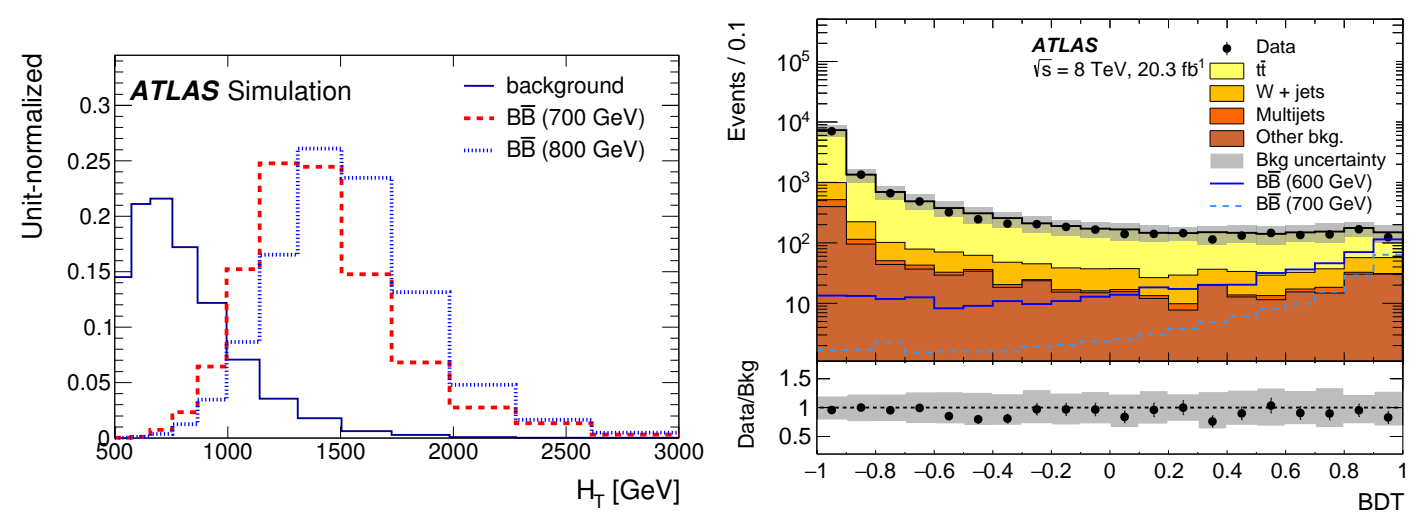

Figure 1: Left: Monte Carlo distributions of $H_{\mathrm{T}}$ for $t \bar{t}$ and $W+$ jets production and vector-like $B \bar{B}$ production for $B$ masses of 700 and $800 \mathrm{GeV}$. Right: Boosted Decision Tree discriminant distributions for data and backgrounds at ATLAS. Both Figures taken from Ref. [6].

Jet substructure techniques have been introduced in several searches in order to take advantage of non-leptonic decays. For instance, CMS has recently updated its $T \bar{T}$ search through its $b W$ decay mode [5]. Hadronic $W$ decays are reconstructed as pairs of "standard" anti- $k_{\mathrm{T}}$ jets [14] with $R=0.5$ as well as single "fat" jets. In the latter case, the "fat" jet is a pruned $[15,16]$ CambridgeAachen jet [17] with $R=0.8, p_{\mathrm{T}}>200 \mathrm{GeV}$, and mass between 60 and $100 \mathrm{GeV}$. The leptonic and hadronic $W$ candidates are paired with other jets, at least one of which is $b$-tagged, with a $\chi^{2}$-like constraint used to prefer pairings with (putative $T \rightarrow b W$ ) masses close to one another. Both $S_{\mathrm{T}}$ and the fitted $b W$ mass are used to discriminate signal from background. Fig. 2(left) shows the data and background distributions in the fitted mass in events with $S_{\mathrm{T}}>1240 \mathrm{GeV}$. A similar analysis has been used in CMS to search for VLQ's of unspecified flavor, $Q \bar{Q} \rightarrow q W q W$, resulting in a preliminary lower mass limit of $788 \mathrm{GeV}$ [18].

The ATLAS search for $Q \bar{Q} \rightarrow q W q W$ also reconstructs hadronic $W$ decays using substructurelike techniques [10]. In this analysis, however, the daughter jets are "standard" ATLAS anti- $k_{\mathrm{T}}$ jets with $R=0.4$, with a requirement that the two jets lie within $\Delta R<1$ of one another, that the combined $p_{\mathrm{T}}$ exceeds $200 \mathrm{GeV}$, the combined mass $m_{12}$ fall between 65 and $100 \mathrm{GeV}$, and the splitting scale $y_{12} \equiv \min \left(p_{\mathrm{T} 1}, p_{\mathrm{T} 2}\right)^{2} \Delta R^{2} / m_{12}^{2}$ [19] is greater than 0.25 . Two $W$ candidates are paired with light-quark jets such that the mass difference between the two pairs is less than $120 \mathrm{GeV}$. The $p_{\mathrm{T}}$ sum of the lepton, missing momentum, and constituent jets is required to exceed $1100 \mathrm{GeV}$. Fig. 2(right) shows the resulting resolved $q W$ mass distribution in data, compared with expected backgrounds and signals with different mass hypotheses and further assuming 100\% branching fractions to $q W$. The data is consistent with the expected background, resulting in the published lower limit on the $Q$ mass at $690 \mathrm{GeV}$ under the $100 \%$ branching fraction assumption. 

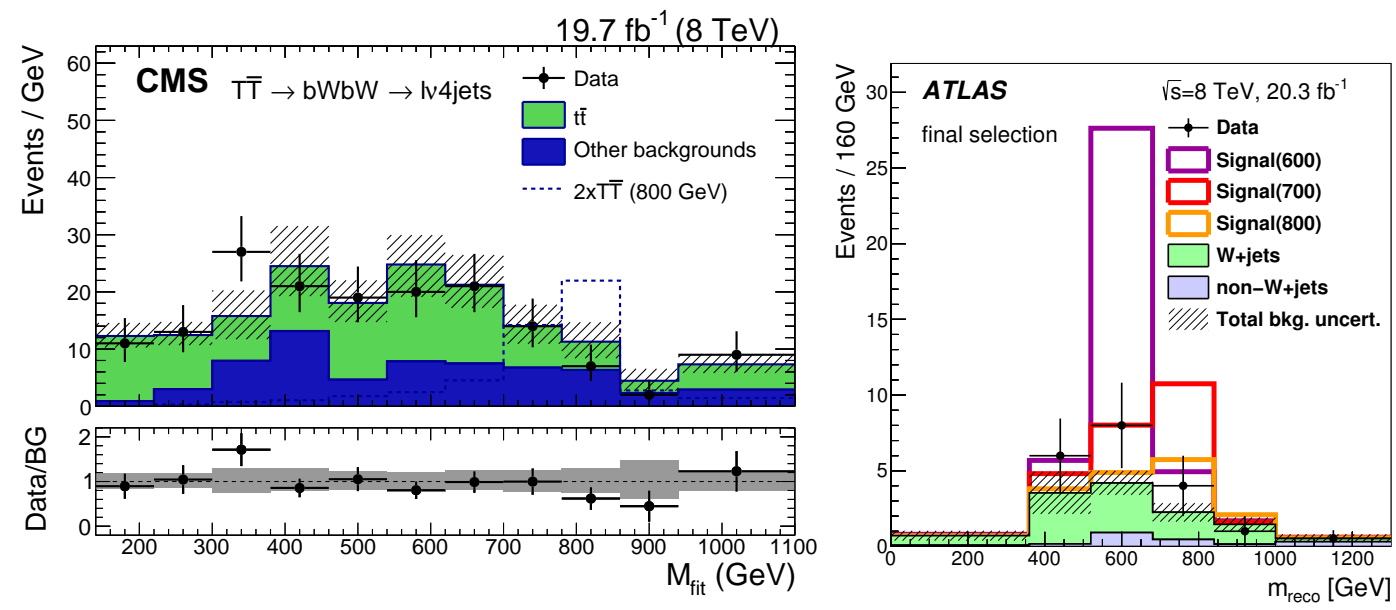

Figure 2: Left: fitted $b W$ mass distributions for the CMS single-lepton $T \bar{T} \rightarrow b W b W$ search. The criterion $S_{\mathrm{T}}>1240 \mathrm{GeV}$ has been applied. Taken from Ref. [5]. Right: resolved $q W$ mass distributions for the ATLAS single-lepton $Q \bar{Q}$ search. Taken from Ref. [10].

\section{Forward single production}

ATLAS has searched for single production of $B$ quarks in the forward (large- $|\eta|$ ) region [20], to complement its earlier search for single $T$ production [8]. The single-lepton search starts with a single high- $p_{\mathrm{T}}$ lepton and missing momentum, and requires two or three standard jets, one of which should be $b$-tagged. The presence of a further high-energy forward jet is required, consistent with the production mechanism. Events with a $W$ or $t$ candidate are tagged by reconstructing trimmed [21] "fat" $(R=1)$ anti- $k_{\mathrm{T}}$ jets with $p_{\mathrm{T}}>200 \mathrm{GeV}$ and $m>50 \mathrm{GeV}$, though the fat jet (which may overlap with the standard jets) plays no further role in the $B$ reconstruction. The visible mass, consisting of the lepton, missing momentum, and the standard jets, is shown in Fig. 3(left) for the case where the lepton comes from the $W$ daughter of the $B$ decay.
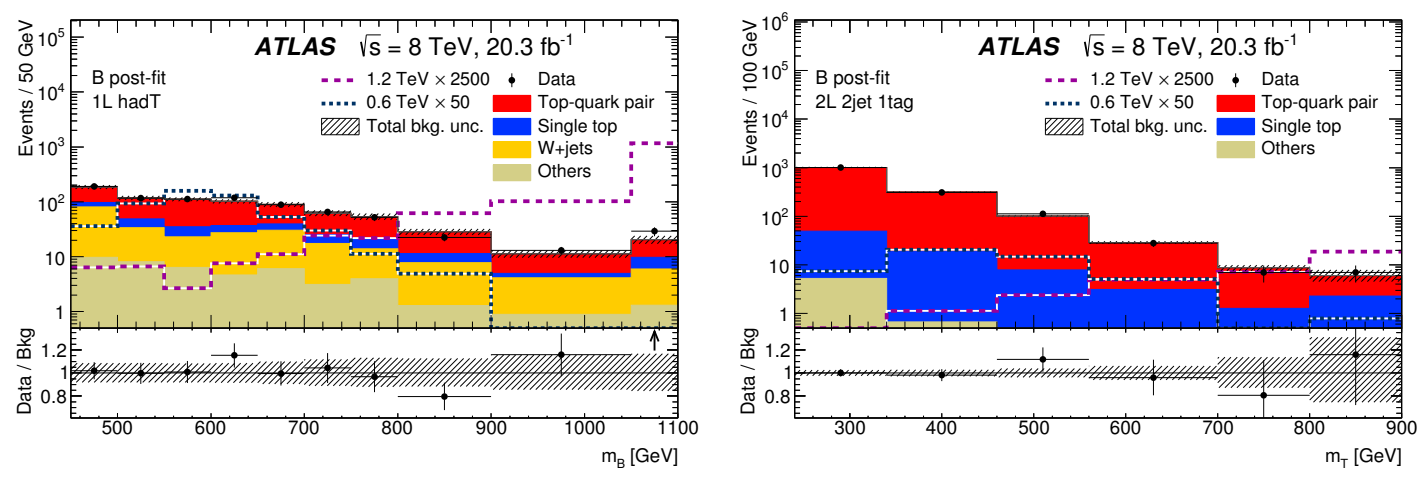

Figure 3: Left: visible mass in ATLAS single $B \rightarrow t W$ search with a single lepton and hadronic $t$ decay. Right: visible mass in $B \rightarrow t W$ search with two leptons. Both Figures taken from Ref. [20].

A search using opposite-sign $e \mu$ pairs is considerably cleaner, and has correspondingly looser requirements. No missing momentum requirement is made, and since both $W$ 's decay leptonically, 
only one central $b$-tagged jet near one of the leptons is required, in addition to the forward jet with $1.5<|\eta|<4.5$. Fig. 3 shows the visible transverse mass distributions. With $20.3 \mathrm{fb}^{-1}$ of data, the search yields an upper limit on the product of the cross section and branching fraction rather than a lower limit on the mass.

\section{Non-leptonic signatures}

CMS has conducted several searches for VLQ's in non-leptonic datasets. The search for $T \bar{T} \rightarrow$ $t H+X$ with $H \rightarrow \gamma \gamma$ takes advantage of the narrow Higgs peak in the diphoton mass spectrum, resulting in a nearly background-free search [5]. The all-hadronic $T \bar{T} \rightarrow b W b W$ search reconstructs two single-jet $W$ 's using pruned fat jets with an additional mass drop criterion, which requires that a splitting is found within the jet where the daughter subjet masses are less than $40 \%$ of the mass of the parent [19]. Opposite $T$ quarks are resolved by requiring a back-to-back topology and a small mass difference between the two $b W$ candidate pairings. The distribution of the scalar sum of the four jets' transverse energy $H_{\mathrm{T}}{ }^{4 \text { jet }}$ is shown in Fig. 4(left). A similar analysis is used to search for $B \bar{B} \rightarrow b H b H$, followed by $H \rightarrow b \bar{b}$ decays.

Both top and Higgs jet tagging are employed on filtered Cambridge-Aachen jets with $R=$ 1.5 in order to search for $T \bar{T} \rightarrow t H t H$ decays [13]. HepTopTAgGER [22], which attempts to distinguish the $t$ decay products inside the fat jet, is used for top tagging, while Higgs tagging looks for two $b$-tagged filtered subjets [19] with mass exceeding $60 \mathrm{GeV}$. The $b \bar{b}$ mass distribution in the best signal region is shown in Fig. 4(right).
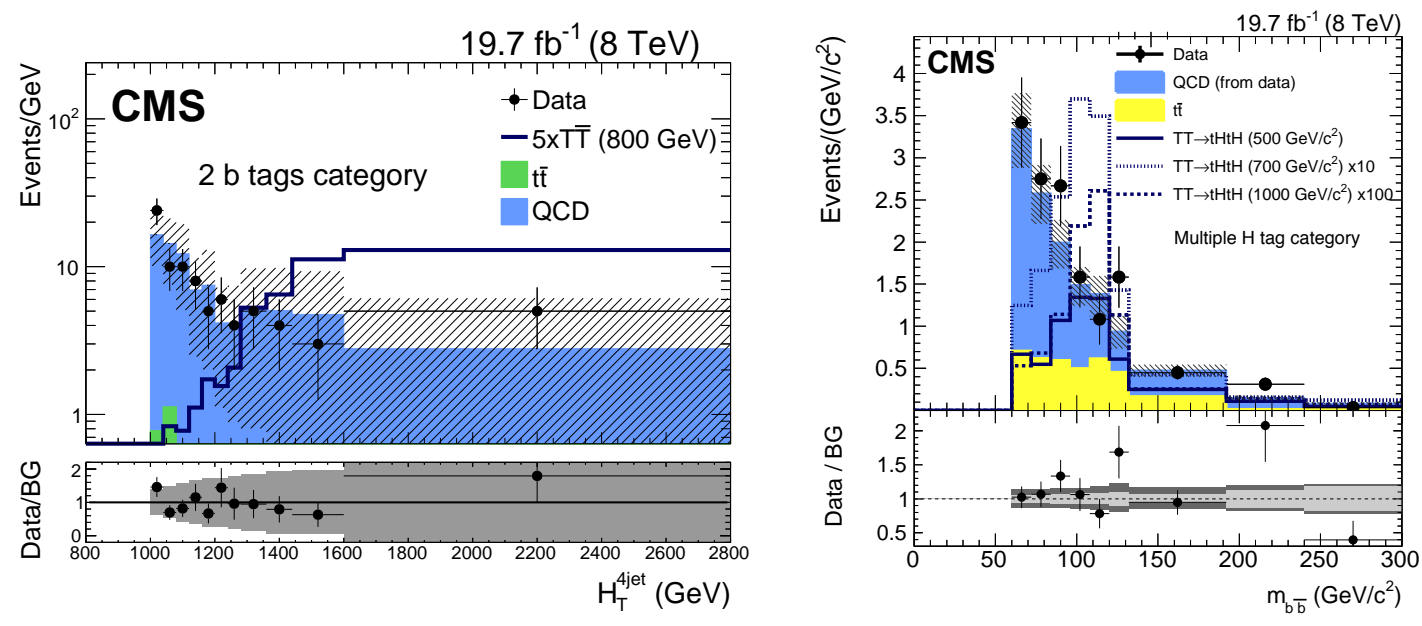

Figure 4: Left: 4-jet $H_{\mathrm{T}}$ distibution for the CMS search for all-hadronic $T \rightarrow b W$ decays. Taken from Ref. [5]. Right: $b \bar{b}$ mass distribution for the CMS search for all-hadronic $T \rightarrow t H$ decays. Taken from Ref. [13].

\section{Mass Limits}

Figures 5 and 6 summarize the 95\% CL lower mass limits for $T$ and $B$ quarks as a function of branching fractions to channels involving $W$ and Higgs bosons, assuming that they couple primarily 
to third-generation Standard Model quarks. Both figures include analyses reported previously and not summarized here. The CMS limits combine the different searches with their systematic uncertainties to obtain Bayesian limits $[4,5]$, while the ATLAS summary plots show the most stringent lower limit, calculated in each analysis using the $\mathrm{CL}_{\mathrm{s}}$ method, for a given set of branching fractions [7]. Within the above assumptions, the current mass limits push above $700 \mathrm{GeV}$ irrespective of model. The single-lepton ATLAS $B \bar{B} \rightarrow t W+X$ search has also yielded the most stringent single mass limit on $T_{5 / 3}$ at $840 \mathrm{GeV}$.
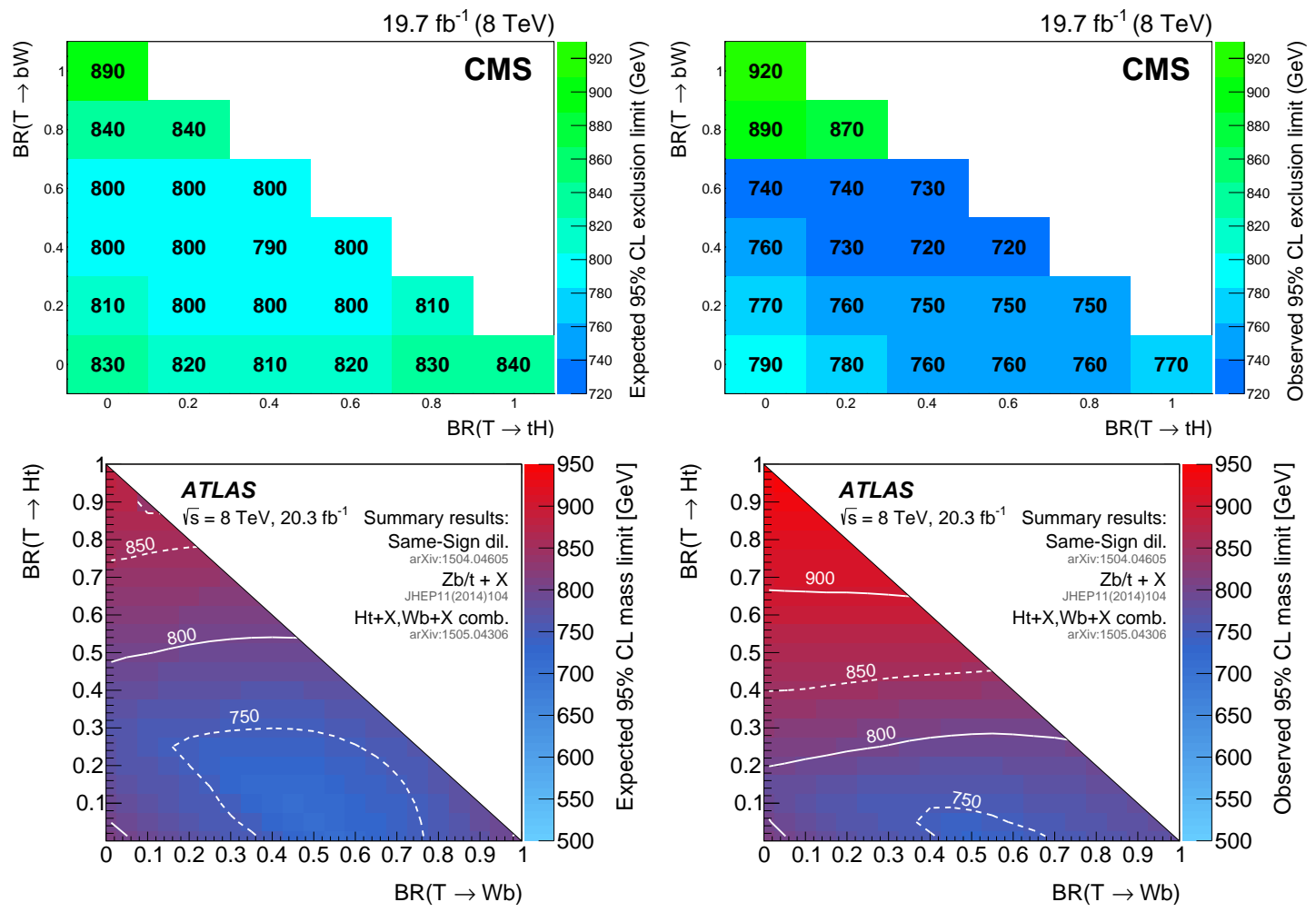

Figure 5: Combined expected (left) and observed (right) 95\% CL lower $T$ mass limits for CMS (top, taken from Ref. [5]) and ATLAS (bottom, taken from Ref. [7]). Note that the axes for the two collaborations are swapped relative to one another.

Both experiments have searched for decay modes involving lighter quark generations, with ATLAS publishing a lower limit of $690 \mathrm{GeV}$ and CMS releasing a preliminary limt of $788 \mathrm{GeV}$, in both cases assuming for the calculation a $100 \%$ branching fraction for $Q \rightarrow q W$. Limits have not been calculated for couplings involving exotic charges, such as $B \rightarrow Z^{\prime} b \rightarrow b \bar{b} b$ [23].

\section{Conclusion}

Vector-like quarks remain a promising signature of physics beyond the Standard Model and arise as a generic feature in a number of classes of such models. ATLAS and CMS have searched for these new particles in roughly $20 \mathrm{fb}^{-1}$ each of data taken at $\sqrt{s}=8 \mathrm{TeV}$ under a number of different model assumptions. No searches have yielded significant excesses, but with both experiments 

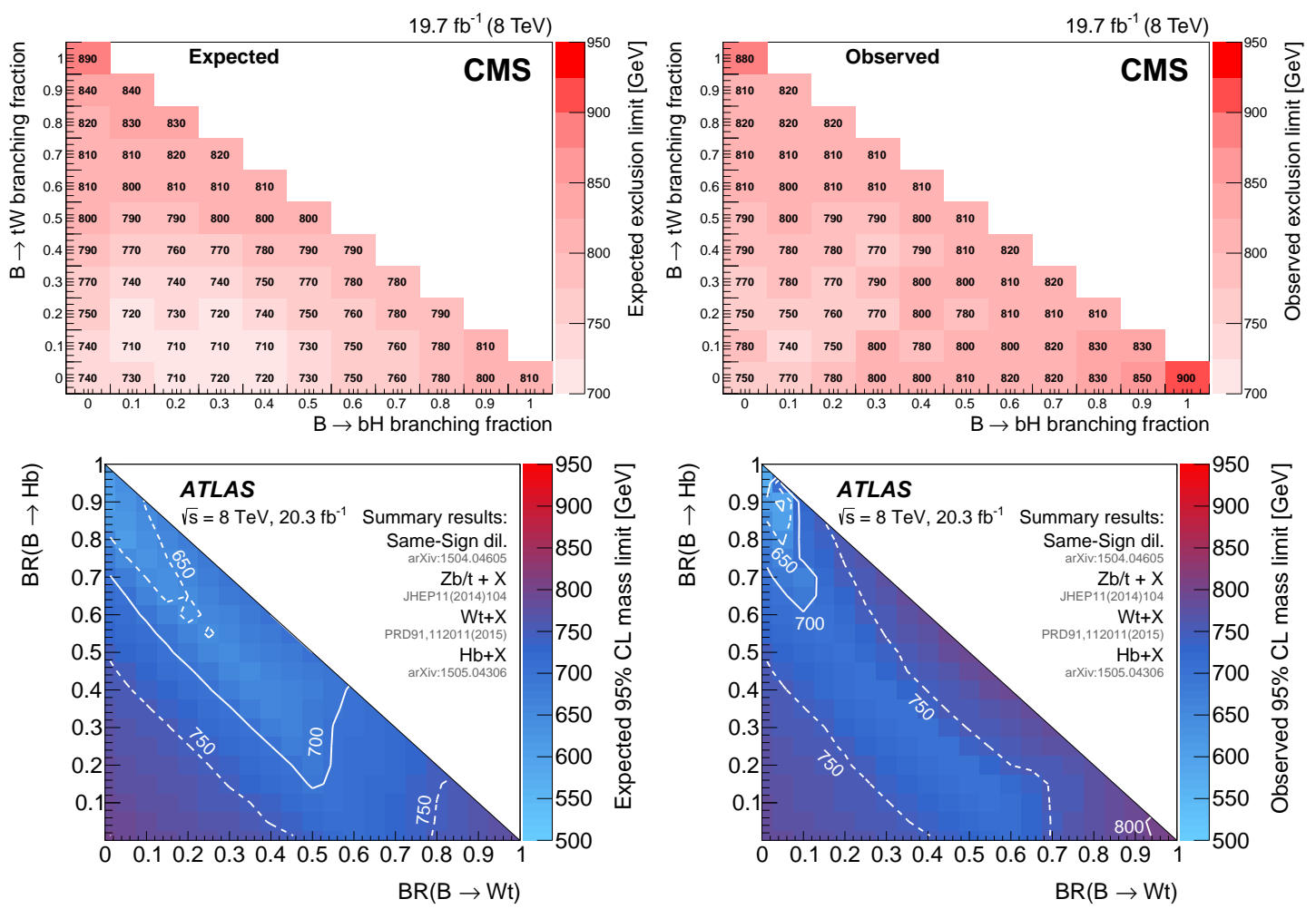

Figure 6: Combined expected (left) and observed (right) 95\% CL lower $B$ mass limits for CMS (top, taken from Ref. [4]) and ATLAS (bottom, taken from Ref. [7]). Note that the axes for the two collaborations are swapped relative to one another.

pursuing and adding new techniques to increase sensitivity, there is eager expectation for data analysis at $13 \mathrm{TeV}$.

The author would like to thank the conference organizers for a congenial and stimulating conference.

\section{References}

[1] ATLAS Collaboration, The ATLAS Experiment at the CERN Large Hadron Collider, JINST 3 (2008) S08003.

[2] CMS Collaboration, The CMS experiment at the CERN LHC, JINST 3 (2008) S08004.

[3] L. Evans and P. Bryant, ed., LHC Machine, JINST 3 (2008) S08001.

[4] CMS Collaboration, Search for pair-produced vector-like B quarks in proton-proton collisions at $\sqrt{s}$ $=8 \mathrm{TeV}$, arXiv:1507.07129 [hep-ex].

[5] CMS Collaboration, Search for Vector-Like Charge 2/3 T Quarks in Proton-Proton Collisions at $\sqrt{s}=$ $8 \mathrm{TeV}$, arXiv:1509.04177 [hep-ex].

[6] ATLAS Collaboration, Search for vectorlike B quarks in events with one isolated lepton, missing transverse momentum and jets at $\sqrt{s}=8 \mathrm{TeV}$ with the ATLAS detector, Phys. Rev. D91 (2015) 112011 [arXiv:1503.05425 hep-ex]. 
[7] ATLAS Collaboration, Search for production of vector-like quark pairs and of four top quarks in the lepton-plus-jets final state in pp collisions at $\sqrt{s}=8 \mathrm{TeV}$ with the ATLAS detector, JHEP 08 (2015) 105 [arXiv:1505.04306 hep-ex].

[8] ATLAS Collaboration, Search for pair and single production of new heavy quarks that decay to a $Z$ boson and a third-generation quark in pp collisions at $\sqrt{s}=8 \mathrm{TeV}$ with the ATLAS detector, JHEP 11 (2014) 104 [arXiv:1409.5500 hep-ex].

[9] ATLAS Collaboration, Analysis of events with b-jets and a pair of leptons of the same charge in pp collisions at $\sqrt{s}=8 \mathrm{TeV}$ with the ATLAS detector, JHEP 10 (2015) 150 [arXiv:1504.04605 hep-ex].

[10] ATLAS Collaboration, Search for pair production of a new heavy quark that decays into a $W$ boson and a light quark in pp collisions at $\sqrt{s}=8 \mathrm{TeV}$ with the ATLAS detector, Phys. Rev. D92 (2015) 112007 [arXiv:1509.04261 hep-ex].

[11] A. Hocker et al., TMVA - Toolkit for Multivariate Data Analysis, POS (ACAT) 040 (2007) [arXiv:physics/0703039].

[12] CMS Collaboration, Inclusive search for a vector-like T quark with charge $\frac{2}{3}$ in pp collisions at $\sqrt{s}=$ 8 TeV, Phys. Lett. B729 (2014) 149-171 [arXiv:1311. 7667 hep-ex].

[13] CMS Collaboration, Search for vector-like T quarks decaying to top quarks and Higgs bosons in the all-hadronic channel using jet substructure, JHEP 06 (2015) 080 [ arXiv: 1503.01952 hep-ex].

[14] M. Cacciari, G. P. Salam, and G. Soyez, The Anti-k(t) jet clustering algorithm, JHEP 04 (2008) 063 [arXiv:0802.1189 hep-ph].

[15] S. D. Ellis, C. K. Vermilion, and J. R. Walsh, Techniques for improved heavy particle searches with jet substructure, Phys. Rev. D80 (2009) 051501 [arXiv:0903.5081 hep-ph] .

[16] S. D. Ellis, C. K. Vermilion, and J. R. Walsh, Recombination Algorithms and Jet Substructure: Pruning as a Tool for Heavy Particle Searches, Phys. Rev. D81 (2010) 094023 [arXiv:0912.0033 hep-ph].

[17] Y. L. Dokshitzer, G. D. Leder, S. Moretti, and B. R. Webber, Better jet clustering algorithms, JHEP 08 (1997) 001 [arXiv:hep-ph/9707323 hep-ph].

[18] CMS Collaboration, Search for vector-like quarks in final states with a single lepton and jets in pp collisions at sqrt $s=8 \mathrm{TeV}$, Tech. Rep. CMS-PAS-B2G-12-017, CERN, Geneva, 2014, http: //cds.cern.ch/record/1976214.

[19] J. M. Butterworth, A. R. Davison, M. Rubin, and G. P. Salam, Jet substructure as a new Higgs search channel at the LHC, Phys. Rev. Lett. 100 (2008) 242001 [arXiv: 0802.2470 hep-ph ] .

[20] ATLAS Collaboration, Search for the production of single vector-like and excited quarks in the Wt final state in pp collisions at $\sqrt{s}=8 \mathrm{TeV}$ with the ATLAS detector, arXiv:1510.02664 [hep-ex].

[21] D. Krohn, J. Thaler, and L.-T. Wang, Jet Trimming, JHEP 02 (2010) 084 [ arXiv: 0912.1342 hep-ph].

[22] T. Plehn and M. Spannowsky, Top Tagging, J. Phys. G39 (2012) 083001 [arXiv: 1112.4441 hep-ph].

[23] P. J. Fox and D. Tucker-Smith, Exotic Decays of Heavy B quarks, arXiv: 1509.00499 [hep-ph]. 